\title{
Application of Mobile Helical Computed Tomography in Combatting COVID-19
}

\author{
Xiaolong Liu (묘 ${ }^{\# 1}$, Zhanguo Sun ${ }^{\# 1}{ }^{\# 1}$, Xiaoqiang Wang ${ }^{1}$, Yueqin Chen ${ }^{1}$, Linsheng Wang ${ }^{1}$, Lizhi Yu ${ }^{2}$, \\ Huiling Yan ${ }^{3}$, Minxia Pang ${ }^{4}$ and Jiehuan Wang ${ }^{1, *}$ \\ ${ }^{1}$ Department of Radiology, Affiliated Hospital of Jining Medical University, Jining, China \\ ${ }^{2}$ Department of Radiology, Taian Municpal Hospital, Taian, China \\ ${ }^{3}$ Department of Emergency, Taian Municpal Hospital, Taian, China \\ ${ }^{4}$ Departemnt of Radiology, Shengli Oilfield Central Hospital, Dongying, China \\ "Corresponding author: Department of Radiology, Affiliated Hospital of Jining Medical University, Jining, China. Email: wjh0112358@163.com \\ \# These authors are contributed equally as the first author.
}

Received 2020 June 08; Revised 2020 December 16; Accepted 2020 December 27.

\section{Abstract}

Background: Chest computed tomography (CT) is a recommended screening and assessment tool for patients with suspected coronavirus disease (COVID-19). However, CT units are currently not available in many temporary hospitals and centralized isolation places.

Objectives: To delineate the workflow of mobile CT unit and evaluate its role in screening for COVID-19 infection in temporary hospitals and centralized isolation locations.

Patients and Methods: Two hundred and twenty-three patients under medical observation in temporary centralized isolation were enrolled in this study. All patients underwent reverse-transcription polymerase chain reaction (RT-PCR) testing and mobile CT chest examinations. Communication, storage, and browsing of CT data were performed with $4 \mathrm{G}$ and cloud technology. Image quality and radiation dose were evaluated and compared with a commercial conventional 64-row CT scanner. Additionally, the sensitivity of initial chest CT and the initial RT-PCR for COVID-19 were compared.

Results: CT examination of 223 patients was completed within 19 work hours. Communication, storage, and browsing of CT data via $4 \mathrm{G}$ and cloud technology were seamless. There were no significant differences in subjective image quality scores between groups (P $>0.05)$. COVID-19 pneumonia was eventually confirmed in 49 patients (21.97\%). The sensitivity of initial chest CT was greater than that of the initial RT-PCR (85.71\% and $67.35 \%$, respectively) $(\mathrm{P}<0.05)$.

Conclusion: Screening suspected patients for COVID-19 by mobile CT in temporary hospitals and isolation points is a simple, efficient, and highly sensitive technique for early diagnosis and control of COVID-19.

Keywords: Coronavirus Disease, Reverse Transcription Polymerase Chain Reaction, Mobile CT, Chest CT Imaging, Diagnostic Value

\section{Background}

Since December 2019, an outbreak of coronavirus disease (COVID-19) has rapidly spread across the world and is a significant threat to human health (1). The numbers of deaths and COVID-19 infections have far exceeded those altogether reported for severe acute respiratory syndrome (774/8098) and Middle East respiratory syndrome (858/2494)(2).

Early diagnosis and isolation are essential to the effective management of COVID-19. Reverse-transcription polymerase chain reaction (RT-PCR) is the gold standard for diagnosis. However, the results of the initial RT-PCR test may be negative in some patients infected with COVID-19. Fang et al. (3) reported that the sensitivity of chest computed tomography (CT) was higher than that of RT-PCR (98\% vs. $71 \%)$. Hence, chest CT is now recommended as an effective screening method for COVID-19 $(4,5)$. CT imaging features of COVID-19, especially bilateral ground-glass opacities or consolidation, have been reported in many articles (5-7).

However, there are still some impediments to the practice of chest CT examination in the context of COVID-19 diagnosis. Many temporary hospitals and centralized isolation places for suspected patients and/or close contacts (e.g., campuses, hotels, stadiums) do not have the equipment necessary to perform CT. Transporting suspected patients and/or close contacts to the hospital for examination is not an optimal solution because of factors including standstill traffic, the potential for hospital cross-infection, 
and limited availability of CT equipment and space at local hospitals. To address these issues, we used a mobile CT unit to screen all relevant personnel. Compared to the conventional CT unit, the mobile CT unit was easy to use and was not as susceptible to environmental factors. Notably, mobile low-dose CT units were previously used to screen for lung cancer (8). However, the use of mobile CT units in the context of a serious public health event has not been reported.

\section{Objectives}

This study delineates the workflow for use of mobile CT units and identifies the value and reliability of this approach in screening for COVID-19 infection.

\section{Patients and Methods}

\subsection{Patients}

The study was approved by the institutional review board and the Centers for Disease Control and Prevention. Signed inform consent was obtained from all study participants.

During the period from February 18 to February 19, 2020, 223 patients under medical observation in temporary centralized isolation facilities with travel/residence history in Wuhan within the past 14 days, history of direct contact with confirmed cases, or unexplained fever and respiratory symptoms were evaluated. All 223 patients underwent RT-PCR testing (GeneoDx Biotech Co, Ltd, Shanghai, China) and chest CT examination with mobile CT on the same day (Figure 1).

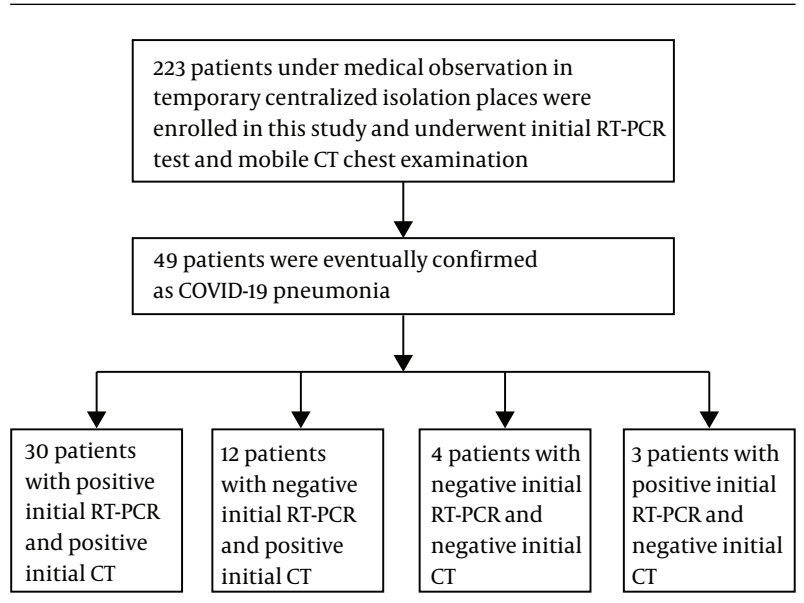

Figure 1. Flowchart of patient inclusion. RT-PCR, reverse-transcription polymerase chain reaction
Two hundred chest CT examinations, examined with a conventional 64-row CT scanner, were enrolled for comparative purposes. These patients were the outpatients who underwent chest CT examination in our hospital during the same period and none of them had a defined history of COVID-19 exposure.

\subsection{CT Data Acquisition, Communication, Storage, and Brows- ing}

The chest CT examinations for the 233 patients included in the study were performed with a mobile 64row whole-body CT scanner (NeuViz 64 In, Neusoft Medical, Shenyang, China), which was transported in a cargo hold that prevented the passage of radiation. CT images were acquired while the patients held their breath, during a single inspiratory phase. The scanning parameters were as follows: tube voltage,120 $\mathrm{kVp}$; tube current, 150 mAs; pitch, 1; helical acquisition mode; detector configuration, $64 \times 0.625 \mathrm{~mm}$; gantry rotation time, $0.6 \mathrm{~s}$; reconstructed section thickness, $5 \mathrm{~mm}$; reconstructed section interval, $5 \mathrm{~mm}$. Lung window images with slice thickness of $1.25 \mathrm{~mm}$ were automatically reconstructed. All CT images were transferred to the Radida cloud-based storage system (Radida Technology Co, Ltd., Beijing, China) via the $4 \mathrm{G}$ network (Long-Term Evolution, China United Network Communication Group Ltd., Beijing, China). Doctors logged in to the Radida cloud-based picture archiving and communication system (PACS) via computer or hand-held device to browse images, compile diagnosis reports, and consult with multidisciplinary teams (Figure 2).

The scanning parameters for conventional chest CT (uCT 780, United Imaging, Shanghai, China) were as follows: tube voltage, $120 \mathrm{kVp}$; tube current, $150 \mathrm{mAs}$; pitch, 1.0875; helical acquisition mode; detector configuration, $64 \times 0.625 \mathrm{~mm}$; gantry rotation time, $0.5 \mathrm{~s}$; reconstructed section thickness, $5 \mathrm{~mm}$; reconstructed section interval, 5 $\mathrm{mm}$. All CT data were transferred to the Vue PACS (Carestream Health, Inc. Toronto, Canada).

\subsection{CT Image Quality and Radiation Dose Evaluation}

For evaluation of imaging quality and radiation dose of the two CTs, 100 CT examinations were respectively randomly selected from patients examined with mobile CT and conventional CT. Data of these 200 CT examinations for evaluation were all transferred to the Vue PACS in an irregular order in advance. All information (device name, scanning settings, data, and patient name) was removed from the display monitors to facilitate blinded evaluation of image quality. Standard mediastinal (window width, $400 \mathrm{HU}$; window level, $40 \mathrm{HU}$ ) and lung parenchymal (window width, $1400 \mathrm{HU}$; window level, -600 HU) window settings were used for image assessment. 


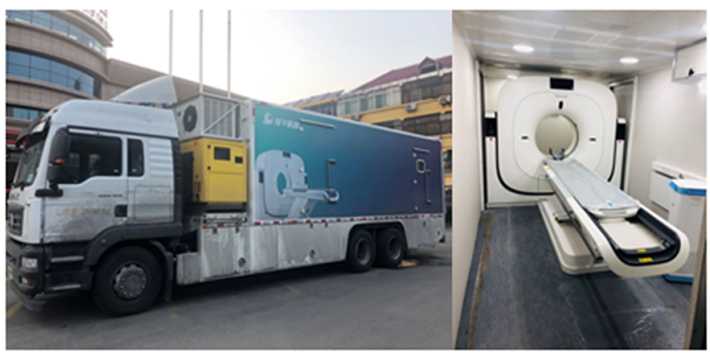

Mobile CT Scanner Unit

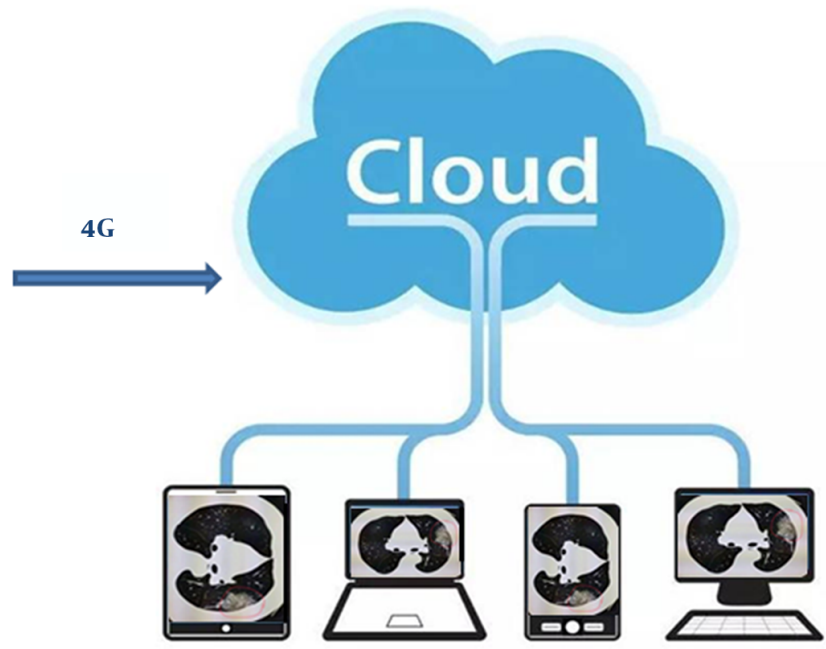

Figure 2. Diagram of the acquisition, communication, storage, and browsing of CT data

The parameters used for the objective evaluation of imaging quality included noise value, signal-to-noise ratio (SNR), and contrast-to-noise ratio (CNR). Circular regions of interest $\left(50 \mathrm{~mm}^{2}\right)$ were drawn by an experienced radiologist on axial images of the lower tracheal air column (at the level $1 \mathrm{~cm}$ above the tracheal bifurcation) and centrally in the lumen of the main pulmonary artery to determine CT attenuation and standard deviation (noise) values (9). For each case, the circular regions of interest were repeatedly drawn and measured three times, and the average measurements were determined. SNR and CNR were determined as described previously in standard operating protocols for the practice of radiology $(9,10)$. The CT dose index of volume (CTDIvol) and dose-length product (DLP) were generated automatically. DLP values were then converted to effective dose (ED) via a universal constant (0.014 $\left.\mathrm{mSv} \times \mathrm{mGy}^{-1} \times \mathrm{cm}^{-1}\right)(11,12)$.

Subjective image evaluation was conducted by two independent radiologists referred to the European guidelines on quality criteria for CT $(13,14)$. The two radiologists were allowed to change the window level, width, and magnification. Image noise, artefacts, visibility of small structures, visually sharp reproduction of chest structures, and diagnostic acceptability of the whole chest imaging were considered. The comprehensive score is 5 , representing 5 quality levels (5, excellent; 4, good; 3, general; 2, defective; 1 , bad). The average value of the two observers was defined as the final subjective quality score. If the difference between the two radiologists' scores was greater than 2 points in the same case, they narrowed the differences through negotiation.

\subsection{Diagnostic Criteria for COVID-19}

According to the preliminary diagnosis and treatment protocols established by the National Health Commission of the People's Republic of China, a diagnosis of COVID-19 is confirmed when viral nucleic acid is detected in throat swabs or in the lower respiratory tract. Positive results on CT were determined according to the CT features previously described for COVD-19 $(7,15,16)$. In patients with initial negative RT-PCR results, the test was routinely repeated before the end of isolation observation. Furthermore, RT-PCR testing would be repeated once more in the wake of typical CT findings of COVID-19, persistence of clinical symptoms or based on patient requirement. Hence, the definitive diagnosis (COVD-19 or not) was based on all PCR results during the 14-day quarantine period.

\subsection{Statistical Analysis}

Data were analyzed by SPSS statistical software, version 17.0 (SPSS Inc., Chicago, Il). Patient characteristics, objective image noise, DLP, ED, SNR, CNR, and image quality scores were displayed as median (interquartile range). Mann-Whitney tests were performed to compare the results obtained by mobile CT with those obtained by conventional CT. For subjective image quality parameters, the extent of agreement between radiologists was determined with Cohen's weighted kappa $(\kappa)$ analysis. The following $\kappa$ values were used to indicate agreement: $0.00-0.20$, poor agreement; 0.21 - 0.40, fair agreement; 0.41 - 0.60, moderate agreement; 0.61 - 0.80, good agreement; 0.81 - 1.00, excellent agreement. Taking the definitive diagnosis as gold standard, sensitivity of the initial RT-PCR and initial chest 
CT in the diagnosis of COVID-19 were compared by the McNemar chi-squared test. P-values $<0.05$ were considered to indicate statistical significance.

\section{Results}

\subsection{Mobile CT Examination}

Chest CT examinations of the 223 patients included in the study were completed within 2 days (19 work hours). The mean examination interval between patients was approximately 5 minutes, with 2 minutes used for disinfection. For each patient, 340 - 460 images were obtained, including a lung window series with section thickness of $5 \mathrm{~mm}$, a lung window series with section thickness of $1.25 \mathrm{~mm}$, and a mediastinal window series (section thickness) of 5mm. Each examination occupied 90 - 110 Mbit. Transmission speed on the $4 \mathrm{G}$ network was $3 \mathrm{Mbit} / \mathrm{s}$. Uploading the results of a single examination to the Radida cloud-based system required $30 \mathrm{~s}$ - $40 \mathrm{~s}$. Episodes of transmission congestion occurred twice, lasting 15 minutes and 25 minutes, respectively. The download speed for cloudbased PACS was $2 \mathrm{Mbit} / \mathrm{s}$. The first time that a clinician browsed an examination, the process required $40 \mathrm{~s}-60 \mathrm{~s}$; however, when using pre-loading function, only approximately $15 \mathrm{~s}$ were required.

\subsection{Image Quality and Dose of Radiation}

One hundred cases who were randomly selected among those examined with mobile CT and another 100 cases who were randomly selected among those examined with conventional CT were reviewed to evaluate patient characteristics, image quality, and radiation dose (Table 1). There were no significant differences between groups in height or weight $(\mathrm{P}>0.05)$. Image noise of the mobile was greater than that of the conventional CT $(\mathrm{P}<0.001)$. SNR, CNR, CTDIvol, DLP, and ED were lower in mobile CT than in conventional CT $(\mathrm{P}<0.001$, respectively). There was good inter-observer agreement for subjective image quality score $(\kappa=0.74)$. The subjective image quality scores did not differ significantly between mobile vs. conventional $\mathrm{CT}(\mathrm{P}>0.05)$. The difference between the two radiologists' scores was not greater than 2 points in any of the cases. For mobile CT scanning, $94 \%$ of the obtained images had scores $\geq 4$.

\subsection{Chest CT and Initial RT-PCR Results}

Of the 223 patients under medical observation (examined with mobile CT), 49 (49/223, 21.97\%) patients were eventually confirmed with COVID-19 pneumonia and hospitalized for treatment. Twelve patients $(12 / 49,24.49 \%)$ initially had negative RT-PCR results but positive chest CT findings indicating viral infection, which appeared as groundglass opacities with or without the crazy-paving sign (Figures 3 and 4). After positive CT findings were obtained, all 12 patients were isolated due to presumed COVID-19 pneumonia. RT-PCR test was repeated every day. Positive results were obtained for three cases on the second day, five cases on the third day, two cases on the fourth day, and one case on the sixth day. In 30 patients (30/49, 61.22\%), the initial results on RT-PCR and CT were positive. The initial results on RT-PCR and CT were both negative in four patients (4/49, $8.16 \%$ ), of whom positive RT-PCR results were obtained on the fourth to seventh day. In three patients (3/49, 6.12\%), the results of chest CT were negative, but the results on RT-PCR were positive. The sensitivity of initial chest CT was greater than that of the initial RT-PCR ( $85.71 \%$ vs. $67.35 \%$, respectively, $\mathrm{P}<0.05$ ).

None of the 200 patients examined with conventional CT were eventually confirmed to have COVID-19 pneumonia.

\section{Discussion}

To delineate the workflow, reliability, and value of mobile CT units in screening for COVID-19 infection, 223 patients under medical observation at temporary centralized isolation facilities were enrolled in the study. Forty-nine patients were eventually confirmed to have COVID-19 pneumonia. The sensitivity of initial chest CT was higher than that of initial RT-PCR, as reported previously $(3,17)$. The reasons for low sensitivity of the initial RT-PCR tests may include use of devices that relied upon rudimentary technology, low patient viral load, or improper clinical sampling (3). Chest CT scanning therefore appears to be a more effective, practical, and more rapid method than RT-PCR for the diagnosis of COVID-19 $(4,18)$. Use of chest CT is therefore recommended as a screening method for patients suspected to have COVID-19.

Given the shortage of CT devices in temporary hospitals and centralized isolation facilities, we explored the feasibility of mobile CT in efforts to screen for COVID-19. CT examinations of the 223 patients were performed successfully with mobile CT over the course of 19 hours. Communication, storage, and browsing of CT data via $4 \mathrm{G}$ and cloud technology proceeded without issue, except for two short-lived low-speed data transmissions due to network congestion. We also evaluated image quality and radiation dose; the results were compared with those obtained via commercial conventional 64-row CT. Given some patients had multiple pulmonary opacities; we drew the region of 


\begin{tabular}{|c|c|c|c|}
\hline Patients' data, image quality and radiation dose & Mobile CT $(\mathrm{N}=100)$ & Conventional CT $(\mathrm{N}=100)$ & $\mathbf{P}$ \\
\hline Height, cm & $170.50(163.25,175.50)$ & $170.00(162.00,175.00)$ & 0.296 \\
\hline Weight, kg & $75(62.00,80.50)$ & $74.5(59.00,80.45)$ & 0.381 \\
\hline Image noise & $9.50(8.30,10.60)$ & $8.29(7.1,9.7)$ & $<0.001$ \\
\hline SNR & $4.46(3.37,4.84)$ & $4.96(4.17,6.09)$ & $<0.001$ \\
\hline CNR & $103.77(90.92,119.49)$ & $122.00(103.46,145.21)$ & $<0.001$ \\
\hline Image quality score & $4.50(4.00,5.00)$ & $4.75(4.00,5.00)$ & 0.16 \\
\hline CTDIvol, mGy & 9.90 & 12.42 & $<0.001$ \\
\hline DLP, mGy/cm & $349.20(330.40,372.77)$ & $430.40(420.44,447.97)$ & $<0.001$ \\
\hline ED, $\mathbf{m S v}$ & $4.89(4.68,5.22)$ & $6.02(5.89,6.27)$ & $<0.001$ \\
\hline
\end{tabular}
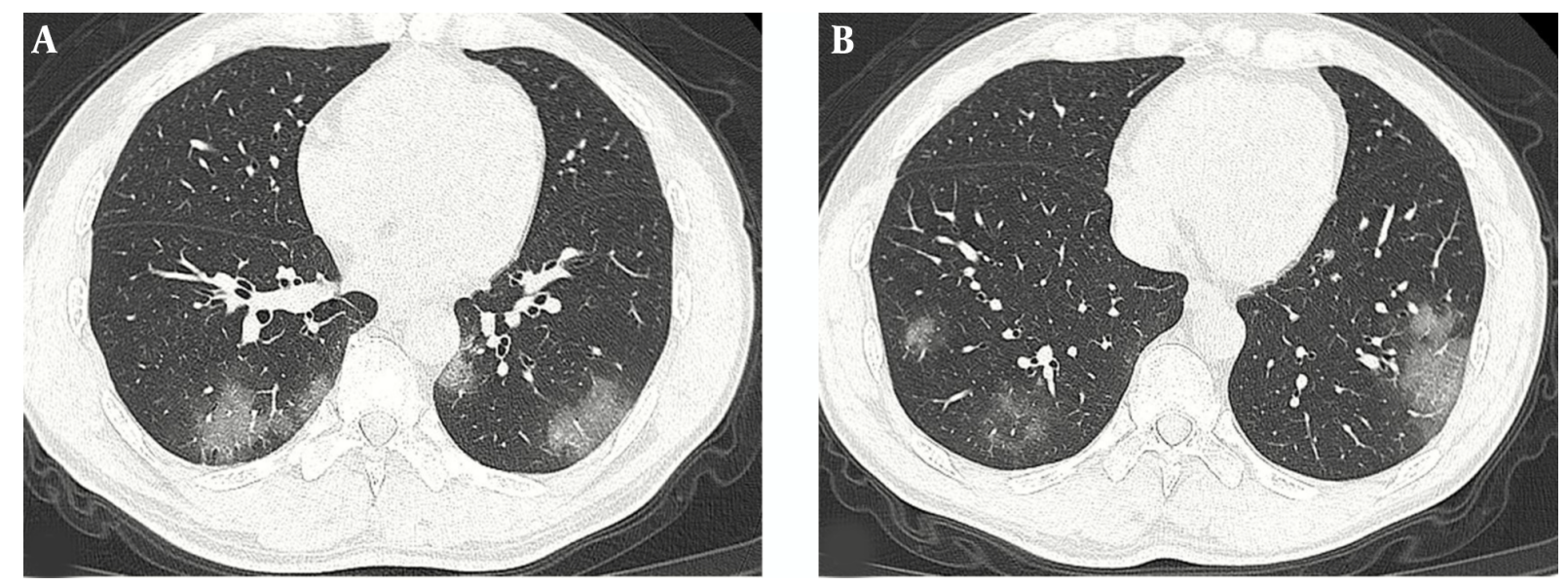

Figure 3. Chest CT images of a 55-year-old man with a history of direct contact with confirmed cases of COVID-19 and a mild fever for 2 days. Axial chest CT revealed bilateral peripheral ground-glass opacities (A, B).

interests (ROIs) for objective imaging quality evaluations on tracheal air column and pulmonary artery by referring the previous article (9). The objective imaging quality of mobile CT was poorer than that of conventional CT in our study. This may be because mobile CT images involved administration of a lower dose of radiation than that administered for conventional CT, as hardware configuration of both CT equipment is similar. Decreasing the radiation dose of CT scanning will lead to the increase of noise and the decrease of SNR and CNR (19). So, image quality is mainly affected by scanning parameters. Besides, during the scanning process, rotation of the tube will cause slight vibration of the machine, which may slightly affect the image quality. However, the subjective image quality scores did not differ significantly between the two CTs. The possi- ble reason is that image quality difference is small in visual sense and does not affect the diagnosis. Overall, mobile CT met the requirements of screening for COVID-19 infection in temporary centralized isolation facilities, in terms of workflow, image quality, and radiation dose.

Mobile CT represents an important advancement in CT technology. Mobile CT units are mainly used for screening (e.g., low-dose CT lung cancer detection). In a study conducted in the United States, Raghavan et al. (8) reported that use of a mobile CT screening unit markedly improved screening rates, with better outcomes at a lower cost per case, in underserved sociodemographic groups. A cargo unit containing a CT scanner and first-aid equipment can also be used as a prehospital mobile stroke unit, which may reduce the time to treatment and increase the rate of intra- 

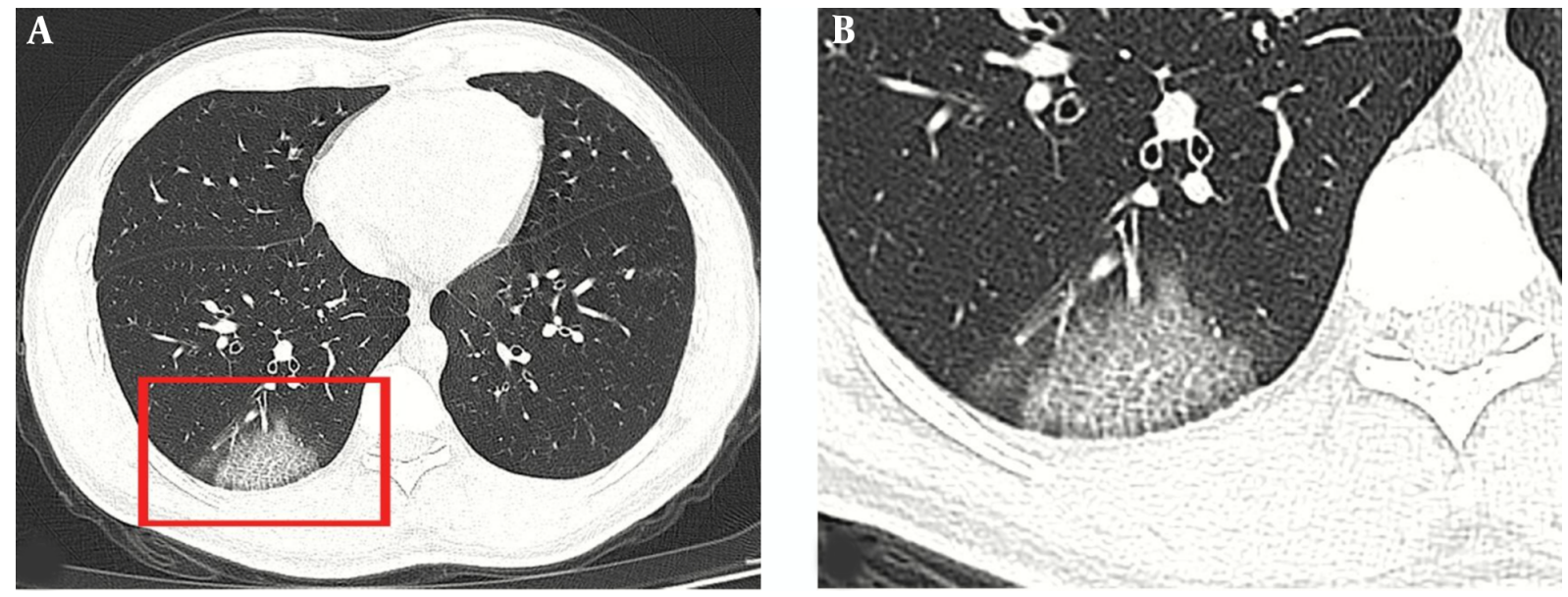

Figure 4. Chest CT images of a 34-year-old man with fever and cough for 3 days. Axial chest CT showed ground-glass opacity with crazy-paving sign (box) in the right lower lobe (A and $B)$.

venous thrombolysis during the "golden hour" (20). Such mobile units can also be used to provide medical aid on the battlefield or in major disaster areas (e.g., neighborhoods affected by earthquake) for the early and timely diagnosis of various types of trauma. Use of such mobile units may thus reduce the number of casualties.

Mobile CT units may have enormous value in the fight against COVID-19. First, the mobile CT unit can move between hospitals and temporary isolation facilities, which maximizes its utilization rate, relieves the shortage of CT equipment, and enables timely diagnosis and treatment. Second, compared to the use of conventional CT in hospitals, use of mobile CT units helps to reduce traffic pressure and may even prevent the cross-infection commonly caused by people moving from one location to another. Third, compared with traditional film and hospital-based PACS, the images stored in the cloud are easy to access at any time and place, and thus more suited for multidisciplinary telemedicine.

Our study provides encouraging results for the use of mobile CT in screening for COVID-19 infection. However, there are some shortcomings to overcome. Efforts to prevent cross-infection are essential throughout the process of examinations. After examination of a given patient, the disposable sheet should be replaced. Disinfectant of machine and floor, properly functioning ventilation, and ultraviolet light are also necessary. Our mobile CT unit supported 5G connections; however, the number of $5 \mathrm{G}$ base stations in the suburb of Jining (Shandong, China) is limited. We therefore chose to use the $4 \mathrm{G}$ connection, which proved stable and reliable. Transmission of CT images was delayed twice due to low transmission speed. When we at- tempted to upload multiple sets of examination results at the same time, congestion resulted. Image transfer is likely to be accelerated with the use of a $5 G$ technology connection, which would prevent network transmission issues. Furthermore, where there is no network coverage, the data may not be uploaded to the cloud PACS immediately. We can choose to upload the data when the network is available. In this case, it is necessary to have an experienced diagnostician on the examination vehicle for timely diagnosis.

Our study had several limitations. This study focused on the use of mobile CT but did not investigate the relationship between CT imaging features and RT-PCR results. Additional studies are therefore needed. Furthermore, this study only roughly evaluated image quality and radiation dose for comparison between mobile CT and conventional CT. The methodology was not as rigorous as possible; image quality as well as radiation dose may be affected by numerous factors that were not investigated in this study.

In conclusion, the results presented above indicate that mobile CT technology is easy to use, efficient, and sensitive for the diagnosis of COVID-19. Mobile CT is useful for screening for COVID-19 among patients suspected to have the disease who are housed in temporary hospitals and isolation facilities. Mobile CT may thus facilitate rapid detection and early isolation. To our knowledge, this is the first report of the application of mobile CT units in the context of serious infectious disease. The empirical results described above may serve as a valuable reference in the fight against COVID-19 and similar serious public health events in the future. 


\section{Footnotes}

Authors' Contributions: Study concept and design: XL, ZS, and JW. Analysis and interpretation of data: XW, LW, LY, MP, and HY. Drafting of the manuscript: XL and ZS. Critical revision of the manuscript for important intellectual content: XL, ZS, and JW. Statistical analysis: YC.

Conflict of Interests: All authors declare that they have no conflict of interest.

Ethical Approval: This study was approved by the Ethics Committee of the Affiliated Hospital of Jining Medical University. The ethical approval code is $2020 \mathrm{C} 013$.

Funding/Support: This work was supported by Jining Key Research and Development Project (No.: 2020YXNS014).

Informed Consent: Written informed consent was obtained from all patients.

\section{References}

1. World Health Organization. Novel Coronavirus (2019-nCoV). 2020, [cited 2020 Jun 6]. Available from: https://www.who.int/ emergencies/diseases/novel-Coronavirus-2019.

2. Zhou S, Wang Y, Zhu T, Xia L. CT Features of Coronavirus Disease 2019 (COVID-19) Pneumonia in 62 Patients in Wuhan, China. AJR Am J Roentgenol. 2020;214(6):1287-94. doi: 10.2214/AJR.20.22975. [PubMed: 32134681].

3. Fang Y, Zhang H, Xie J, Lin M, Ying L, Pang P, et al. Sensitivity of Chest CT for COVID-19: Comparison to RT-PCR. Radiology. 2020;296(2):E1157. doi: 10.1148/radiol.2020200432. [PubMed: 32073353]. [PubMed Central: PMC7233365].

4. Kim H. Outbreak of novel coronavirus (COVID-19): What is the role of radiologists? Eur Radiol. 2020;30(6):3266-7. doi: 10.1007/s00330-02006748-2. [PubMed: 32072255]. [PubMed Central: PMC7087878].

5. Ai T, Yang Z, Hou H, Zhan C, Chen C, Lv W, et al. Correlation of Chest CT and RT-PCR Testing for Coronavirus Disease 2019 (COVID19) in China: A Report of 1014 Cases. Radiology. 2020;296(2):E32-40. doi: 10.1148/radiol.2020200642. [PubMed: 32101510]. [PubMed Central: PMC7233399].

6. Shi H, Han X, Jiang N, Cao Y, Alwalid O, Gu J, et al. Radiological findings from 81 patients with COVID-19 pneumonia in Wuhan, China: a descriptive study. Lancet Infect Dis. 2020;20(4):425-34. doi: 10.1016/S1473-3099(20)30086-4. [PubMed: 32105637]. [PubMed Central: PMC7159053].

7. Chung M, Bernheim A, Mei X, Zhang N, Huang M, Zeng X, et al. CT Imaging Features of 2019 Novel Coronavirus (2019-nCoV). Radiology. 2020;295(1):202-7. doi: 10.1148/radiol.2020200230. [PubMed: 32017661]. [PubMed Central: PMC7194022].

8. Raghavan D, Wheeler M, Doege D, Doty J2, Levy H, Dungan KA, et al. Initial Results from Mobile Low-Dose Computerized Tomographic Lung
Cancer Screening Unit: Improved Outcomes for Underserved Populations. Oncologist. 2020;25(5):e777-81. doi: 10.1634/theoncologist.20190802. [PubMed: 31771991]. [PubMed Central: PMC7216453].

9. Braun FM, Johnson TR, Sommer WH, Thierfelder KM, Meinel FG. Chest CT using spectral filtration: radiation dose, image quality, and spectrum of clinical utility. Eur Radiol. 2015;25(6):1598-606. doi: 10.1007/s00330-014-3559-1. [PubMed: 25515204].

10. Symons R, Pourmorteza A, Sandfort V, Ahlman MA, Cropper T, Mallek $\mathrm{M}$, et al. Feasibility of Dose-reduced Chest CT with Photon-counting Detectors: Initial Results in Humans. Radiology. 2017;285(3):980-9. doi: 10.1148/radiol.2017162587. [PubMed: 28753389]. [PubMed Central: PMC5708286].

11. Shrimpton PC, Hillier MC, Lewis MA, Dunn M. National survey of doses from CT in the UK: 2003. Br J Radiol. 2006;79(948):968-80. doi: 10.1259/bjr/93277434. [PubMed: 17213302].

12. Messerli M, Giannopoulos AA, Leschka S, Warschkow R, Wildermuth S, Hechelhammer L, et al. Diagnostic accuracy of chest Xray dose-equivalent CT for assessing calcified atherosclerotic burden of the thoracic aorta. Br J Radiol. 2017;90(1080):20170469. doi: 10.1259/bjr.20170469. [PubMed: 28972810]. [PubMed Central: PMC6047658].

13. Singh S, Kalra MK, Gilman MD, Hsieh J, Pien HH, Digumarthy SR, et al. Adaptive statistical iterative reconstruction technique for radiation dose reduction in chest CT: a pilot study. Radiology. 2011;259(2):56573. doi: 10.1148/radiol.11101450. [PubMed: 21386048].

14. Singh S, Kalra MK, Shenoy-Bhangle AS, Saini A, Gervais DA, Westra $S J$, et al. Radiation dose reduction with hybrid iterative reconstruction for pediatric CT. Radiology. 2012;263(2):537-46. doi: 10.1148/radiol.12110268. [PubMed: 22517962].

15. Duan YN, Qin J. Pre- and Posttreatment Chest CT Findings: 2019 Novel Coronavirus (2019-nCoV) Pneumonia. Radiology. 2020;295(1):21. doi: 10.1148/radiol.2020200323. [PubMed: 32049602]. [PubMed Central: PMC7233361].

16. Pan Y, Guan H. Imaging changes in patients with 2019-nCov. Eur Radiol. 2020;30(7):3612-3. doi: 10.1007/s00330-020-06713-z. [PubMed: 32025790]. [PubMed Central: PMC7075276].

17. Xie X, Zhong Z, Zhao W, Zheng C, Wang F, Liu J. Chest CT for Typical Coronavirus Disease 2019 (COVID-19) Pneumonia: Relationship to Negative RT-PCR Testing. Radiology. 2020;296(2):E41-5. doi: 10.1148/radiol.2020200343. [PubMed: 32049601]. [PubMed Central: PMC7233363].

18. Kanne JP. Chest CT Findings in 2019 Novel Coronavirus (2019-nCoV) Infections from Wuhan, China: Key Points for the Radiologist. Radiology. 2020;295(1):16-7. doi: 10.1148/radiol.2020200241. [PubMed: 32017662]. [PubMed Central: PMC7233362].

19. Chen EL, Ross JA, Grant C, Wilbur A, Mehta N, Hart E, et al. Improved Image Quality of Low-Dose CT Pulmonary Angiograms. J Am Coll Radiol. 2017;14(5):648-53. doi: 10.1016/j.jacr.2016.11.007. [PubMed: 28082157].

20. Nyberg EM, Cox JR, Kowalski RG, Vela-Duarte D, Schimpf B, Jones WJ. Mobile Stroke Unit Reduces Time to Image Acquisition and Reporting. AJNR Am J Neuroradiol. 2018;39(7):1293-5. doi: 10.3174/ajnr.A5673. [PubMed: 29773569]. [PubMed Central: PMC7655441]. 